\title{
Changing Vivax Malaria Transmission Features in the Republic of Korea during 2013-2014
}

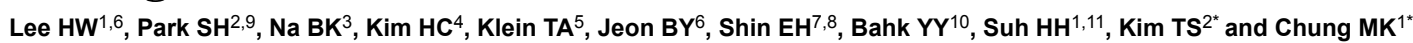

${ }^{1}$ Institute of Research and Development, CELLBios Inc., Seoul, 08507, Republic of Korea

${ }^{2}$ Department of Tropical Medicine, Inha University School of Medicine, Incheon 22212, Republic of Korea

${ }^{3}$ Department of Parasitology and Institute of Health Sciences, Gyeongsang National University School of Medicine, Jinju 660-751, Republic of Korea

${ }^{4} 5$ th Medical Detachment, 168th Multifunctional Medical Battalion, 65th Medical Brigade, Unit 15247, APO AP 96205-5247, USA

565th Medical Brigade, Unit 15281, APO AP 96205-5281, USA

${ }^{6}$ Department of Biomedical Laboratory Science, College of Health Sciences, Yonsei University, School of Public Health, Wonju, 26493, Republic of Korea

${ }^{7}$ Department of Parasitology and Tropical Medicine, and Institute of Endemic Diseases, Seoul National University College of Medicine, Seoul 110-799, Republic of Korea

${ }^{8}$ Seoul National University Bundang Hospital, Seongnam 463-707, Republic of Korea

${ }^{9}$ Department of Hygienic Research, Incheon Metropolitan City Public Health and Environment Research Institute, Incheon, 22320, Republic of Korea

${ }^{10}$ Department of Biotechnology, College of Biomedical and Health Science, Konkuk University, Chungju, 27478, Republic of Korea

${ }^{11}$ Department of Environmental Engineering, Gyeongnam National University of Science and Technology, Jinju, 52725, Republic of Korea

*Corresponding authors: Tong-Soo Kim, Department of Tropical Medicine, Inha University School of Medicine, Incheon 22212, Republic of Korea, Tel: +82-010-8621-2676; Fax: +82-032-885-8302; E-mail: tongsookim@inha.ac.kr

Mi-Kyung Chung, Institute of Research and Development, CELLBios Inc., Seoul, 08507, Republic of Korea, Tel:+82-2-838-0803, Fax: +82-2-838-0804, E-mail: mikychung@daum.net

Received date: February 02, 2017; Accepted date: February 08, 2017; Published date: February 18, 2017

Copyright: (c) 2017 Lee HW, et al. This is an open-access article distributed under the terms of the Creative Commons Attribution License, which permits unrestricted use, distribution, and reproduction in any medium, provided the original author and source are credited.

\begin{abstract}
Since the re-emergence of Plasmodium vivax in the Republic of Korea (ROK), 32,197 cases of vivax malaria have been reported (1993-2014). The aim of this study was to review the notification records of malaria to the Korean Centres for Disease Control and Prevention to investigate its transmission during the period 2013-2014. Reporting of malaria cases confirmed by microscopic examination is mandatory in the ROK. In this study, all available records of malaria cases and collected malaria vectors from eight locations in Gangwon Province, Gyeonggi Province, and Incheon Metropolitan City during 2013-2014 were reviewed. During the study period, 943 malaria cases were recorded. The number of cases reported in 2013 decreased by $30.6 \%$ (385 cases) compared with that in 2012 (555 cases); however, it increased by 44.9\% in 2014 (558 cases) compared with that in 2013. This change may be due to the increased malaria vector density. Anopheles sinensis sensu lato increased at most collection sites in 2014 compared with that in 2013. In addition, the annual mean temperature and precipitation were slightly increased in 2014 compared with those in 2013.
\end{abstract}

An increase in the number of malaria cases in a short period requires the attention of the officer in charge in order to treat the disease. More-intensive surveillance is needed in high-risk areas to identify the factors that affect the incidence of malaria in the ROK.

Keywords: Plasmodium vivax, Anopheles sinensis, Transmission; Re-emerging

\section{Introduction}

Plasmodium vivax is the causative agent of relapsing human malaria, the second most common type in humans. Vivax malaria afflicts several hundred million people annually worldwide, and it is a major public health problem in many tropical, semitropical, and temperate countries, including North and South Korea [1].

Malaria has been prevalent throughout the Korean peninsula for a long time, with the first scientific report of Korean vivax malaria published in 1913 [2].

As a result of the national malaria eradication program conducted in cooperation with the World Health Organization (WHO), the vivax malaria cases in the Republic of Korea (ROK) rapidly decreased; in 1979, the WHO declared that malaria was eradicated in the ROK [3-5].

After two sporadic cases detected in the 1980s [6], a ROK soldier who was stationed in the northern Gyeonggi Province in 1993 [7] and two patients in 1994 were diagnosed with vivax malaria [8]. Since then, a number of malaria cases have been reported near the demilitarized zone (DMZ).

During the first few years after the re-emergence of malaria in Korea, most cases occurred in Korean army personnel deployed or while on leave near the DMZ.

As the total number of malaria cases increased, the ratio of civilianto-army cases also increased [9]. 
A considerable concern emerged that malaria might be transmitted throughout Korea through veteran soldiers returning to their hometowns [10-12].

The purpose of this study was to analyze the malaria transmission in Korea by determining the annual number of patients (army soldiers, veterans, and civilians), the geographic distribution of cases, and the density of Anopheles mosquitoes captured using black-light traps in the malaria-risk areas during 2013-2014.

\section{Materials and Methods}

\section{Data collection}

The Korean government is systematically attempting to control malaria, which is classified as a group III communicable disease. Malaria patients were categorized as civilians, veterans, or army soldiers for the convenience of data analysis. For civilians and veterans, cases of malaria detected in private hospitals or clinics have to be reported to the local public health center (PHC).

The PHC should report the data periodically to the Division of Infectious Disease Surveillance (DIDS) at the Korean Centres for Disease Control and Prevention (KCDC). For army soldiers, cases should be reported to the Office of the Surgeon General, Army Headquarters, and the Ministry of Health and Welfare [13]. These reports should have the patient's name, date of onset of illness, date of diagnosis, age, sex, address, and the estimated areas where the infection occurred. Most malaria cases, including those of veterans, were diagnosed in civilian hospitals or health clinics, and might also include army soldiers who were diagnosed while on leave.

However, such cases in army soldiers are excluded from the civilian/ veteran cases by the DIDS during the verification process $[14,15]$.

After obtaining approval from the Institutional Review Board of the Korean National Institute of Health, the records submitted to the KCDC and trends based on patients' sex, age, and occupation were analyzed without revealing patient identity.

\section{Definition of malaria}

Thick and thin blood films of samples from patients with suspected malaria were fixed with methanol and stained with Giemsa solution according to standard procedures [16].

To estimate the density of blood-stage parasites, the number of parasites identified in 200 white blood cells (WBCs) was counted, and the parasite-to-WBC ratio was multiplied by 8,000 [17].

\section{Geographic distribution of the patients with malaria}

The annual geographic distribution of malaria cases in civilians, veterans, and army soldiers was determined based on the patient's residences and travel histories. Seasonal incidence was analyzed by grouping cases at weekly or monthly intervals.

\section{Calculation of annual parasite incidence}

The annual parasite incidence (API) was calculated as the number of malaria-positive patients per 100,000 inhabitants for each study area; that is, $\mathrm{API}=$ (number of microscopically proven malaria cases/ population of administrative area) $\times 100,000$.

\section{Density of Anopheles mosquitoes}

The number of Anopheles mosquitoes was monitored using blacklight traps in Gyodong-myeon (Figure 1A) and Wolgot-ri (Figure 1A) in Ganghwa County of Incheon Metropolitan City, Sau-dong of Gimpo-si (Figure 1B) and Daesung-dong of Paju-si (Figure 1B) in Gyeonggi Province, Daema-ri (Figure 1C) and Haksa-ri (Figure 1C) of Cheorwon County, and Guam-ri (Figure 1C) and Myeongpa-ri (Figure 1C) in Gangwon Province in the ROK during the malaria transmission seasons from 2013 to 2014.

Mosquito collections were conducted twice a week between 2000 and 0700 hours from April (14th week) to October (44th week) at the same collection sites for comparative analysis. Female mosquitoes were identified on the basis of morphological differences and were counted regardless of blood-fed status or malaria parasite infectivity. The average number of collected mosquitoes over a 2-day period was considered a representative value for the week [14].

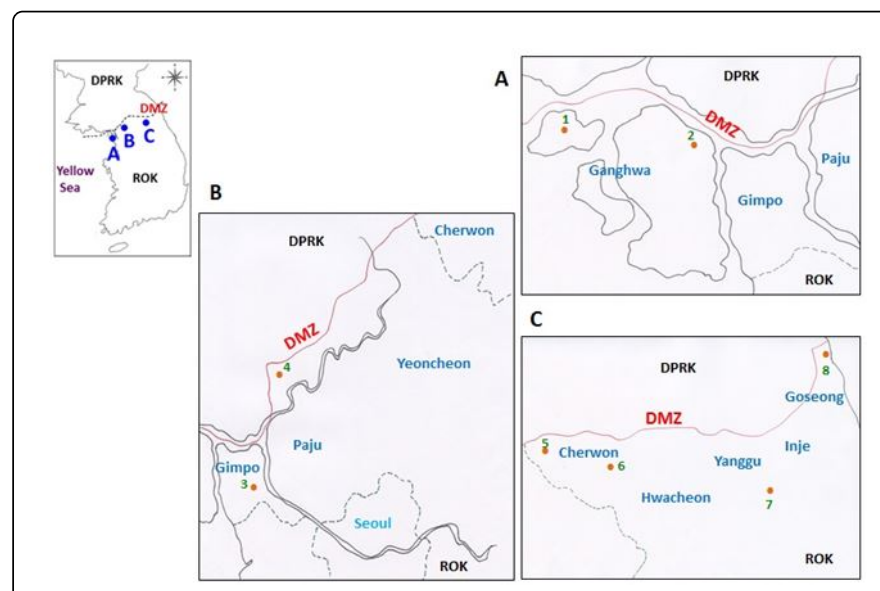

Figure 1: Locations where mosquitoes were collected with black light traps. A, Incheon Metropolitan City; B, Gyeonggi Province; C, Gangwon Province; 1, Gyodong-myeon; 2, Wolgot-ri; 3, Sau-dong; 4, Daesung-dong; 5, Daema-ri; 6, Haksa-ri; 7, Guam-ri; 8, Myeongpa-ri.

\section{Results}

During the 20 years after the reemergence of malaria in the ROK (1993-2014), 32,197 cases of vivax malaria were reported (Figure 2); 9,696 cases $(30.1 \%)$ were reported in ROK military soldiers, 7,777 cases $(24.2 \%)$ were reported in veterans who served in malaria highrisk areas, and 14,724 cases (45.7\%) were reported in civilians.

During this period, the incidence of malaria peaked at three time points, in 2000, 2007, and 2010. Until 2002, the number of cases in military-related personnel, soldiers, and veterans was higher than that in civilians. After 2002, the number of civilian cases was much higher than that of military-related cases, except in 2009 and 2011.

Comparison of the number of civilian cases with that of soldier cases only indicated that the civilian cases exceeded the soldier cases after 1999. From 2013 to 2014, 943 vivax malaria cases were reported, including 195 cases (20.7\%) in soldiers, 119 (12.6\%) in veterans, and $629(66.7 \%)$ in civilians. The number of cases reported in Seoul Metropolitan City, Incheon Metropolitan City, and Gyeonggi Province increased by $105.4 \%, 57.7 \%$, and $41.7 \%$, respectively, in 2014 . However, 
Citation: $\quad$ Lee HW, Park SH, Na BK, Kim HC, Klein TA, Jeon BY, Shin EH, Bahk YY, Suh HH, Kim TS* and Chung MK* (2017) Changing Vivax Malaria Transmission Features in the Republic of Korea during 2013-2014. J Trop Dis 5: 231. doi:10.4172/2329-891X.1000231

Page 3 of 9

in Gangwon Province, the number of cases decreased by $40.0 \%$, from 25 cases in 2013 to 15 cases in 2014 (Table 1).

In addition, the US military reported 14 malaria cases during 2013-2014. Nine cases were reported in personnel currently deployed in Korea (one case in 2013 and eight cases in 2014), and five cases, two cases in 2013 and three cases in 2014 (unpublished data), were detected in US military personnel after their return to the United States, possibly owing to the long incubation period of vivax malaria. The second highest peak was observed in the 39th week of 2013 (September 22-28; 24 patients) after Chuseok (Korean Thanksgiving Day, September 19). Several more peaks were observed during the transmission season; in 2014, another peak was observed in the 38th week (September 14-20), again after Chuseok (September 8), with 27 patients. Interestingly, in the 41st week (early October) in both 2013 and 2014, the last peak of the transmission season was observed (15 patients in 2013 and 19 patients in 2014).

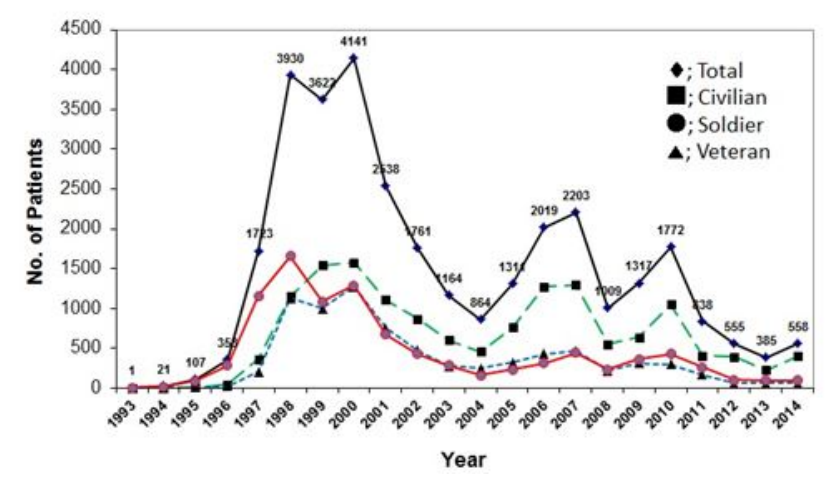

Figure 2: The annual number of vivax malaria cases from 1993 to 2014.
In addition, the US military reported 14 malaria cases during 2013-2014. Nine cases were reported in personnel currently deployed in Korea (one case in 2013 and eight cases in 2014), and five cases, two cases in 2013 and three cases in 2014 (unpublished data), were detected in US military personnel after their return to the United States, possibly owing to the long incubation period of vivax malaria.

Overall, the API decreased from 1.08 in 2012 to 0.75 in 2013 and then increased to 1.09 in 2014. In 2012, Incheon Metropolitan City had the highest API (4.93), followed by Gyeonggi Province (2.13), Gangwon Province (0.87), and Seoul Metropolitan City.

In Gangwon Province, it was first detected during the 24th week of 2013 and the 19th week of 2014 in Daema-ri, Cheorwon County; during the 24th week of 2013 and the 15th week of 2014 in Haksa-ri, Cheorwon County; during the 20th week of 2013 and the 19th week of 2014 in Guam-ri, Yanggu County; and during the 24th week of 2013 and the 23rd week of 2014 in Myeongpa-ri, Gosung County.

US military reported 14 malaria cases during 2013-2014. Nine cases were reported in personnel currently deployed in Korea (one case in 2013 and eight cases in 2014), and five cases. Two cases in 2013 and three cases in 2014 (unpublished data), were detected in US military personnel after their return to the United States, possibly owing to the long incubation period of vivax malaria.

The proportion of civilian cases increased from 59.0\% in 2013 to $72.0 \%$ in 2014 , while the proportion of ROK cases in soldiers and veterans decreased from $97(25.2 \%)$ in 2013 to $98(17.6 \%)$ in 2014, respectively (Figure 3 ).

\begin{tabular}{|c|c|c|c|c|c|}
\hline Area & Malaria incidence & Malaria incidence & Percent Difference & Malaria incidence & Percent Difference \\
\hline $\begin{array}{l}\text { Metro- } \\
\text { politan Areas }\end{array}$ & 2012 & 2013 & 2013 & 2014 & 2014 \\
\hline Seoul & 67 & 37 & -55.2 & 76 & +105.4 \\
\hline Busan & 7 & 3 & -57.1 & 7 & +133.3 \\
\hline Daegu & 4 & 4 & 0.0 & 3 & -25.0 \\
\hline Incheon & 143 & 78 & -45.5 & 123 & +57.7 \\
\hline Gwangju & 1 & 3 & +200.0 & 3 & 0.0 \\
\hline Daejeon & 4 & 2 & -50.0 & 1 & -50.0 \\
\hline Ulsan & 3 & 1 & -66.7 & 2 & +100.0 \\
\hline $\begin{array}{l}\text { Provinces } \\
\text { Gyeonggi }\end{array}$ & 263 & 204 & -22.4 & 289 & +41.7 \\
\hline Gangwon & 13 & 25 & +92.3 & 15 & -40.0 \\
\hline Chungbuk & 8 & 3 & -62.5 & 3 & 0.0 \\
\hline Chungnam & 7 & 2 & -71.4 & 9 & +350.0 \\
\hline
\end{tabular}


Citation: $\quad$ Lee HW, Park SH, Na BK, Kim HC, Klein TA, Jeon BY, Shin EH, Bahk YY, Suh HH, Kim TS* and Chung MK* (2017) Changing Vivax Malaria Transmission Features in the Republic of Korea during 2013-2014. J Trop Dis 5: 231. doi:10.4172/2329-891X.1000231

Page 4 of 9

\begin{tabular}{|l|l|l|l|l|l|}
\hline Jeonbuk & 5 & 2 & -60.0 & 8 & +300.0 \\
\hline Jeonnam & 4 & 3 & -25.0 & 1 & -66.7 \\
\hline Gyeongbuk & 8 & 11 & +37.5 & 9 & -18.2 \\
\hline Gyeongnam & 16 & 5 & -68.8 & 8 & +60.0 \\
\hline Jeju & 2 & 2 & 0.0 & 1 & -50.0 \\
\hline Total & 555 & 385 & -30.6 & 558 & +44.9 \\
\hline
\end{tabular}

Table 1 : Annual number and percent difference of Plasmodium vivax cases by Metropolitan City and Province, 2012-2014.

PHCs have the responsibility to control the transmission of malaria in each risk area. As a result of anti-malaria efforts by the PHC in Cheorwon County where there is a high-risk area in Gangwon Province, the number of malaria cases in 2014 decreased from 25 cases in 2013 (API: 1.67) to 15 cases (API: 1.00), which is close to same number of cases documented in 2012 (13 cases; API: 0.87; Figure 4).

Interestingly, this province showed a reverse pattern in case numbers compared with that observed in other metropolitan cities and provinces (Incheon, Seoul, and Gyeonggi), where the numbers decreased in 2013 and increased in 2014 (Table 1).

This may be because of the average duration until confirmation of suspected malaria cases, which was 5.12 days in 2014 but longer than that (7 days) in 2013 (unpublished data).

The anti-mosquito treatments, including fogging and residual spraying, were performed by the PHC in Cheorwon County from May 1 to the end of October in both 2013 and 2014 (unpublished data).

Larvicide was used eight times in 2013 and 10 times in 2014. Insecticide was applied 632 times in 2013 and 674 times in 2014 to cover an area of $36,592 \mathrm{~m}^{2}$ (data from the PHC in Cheorwon County, Gangwon Province).

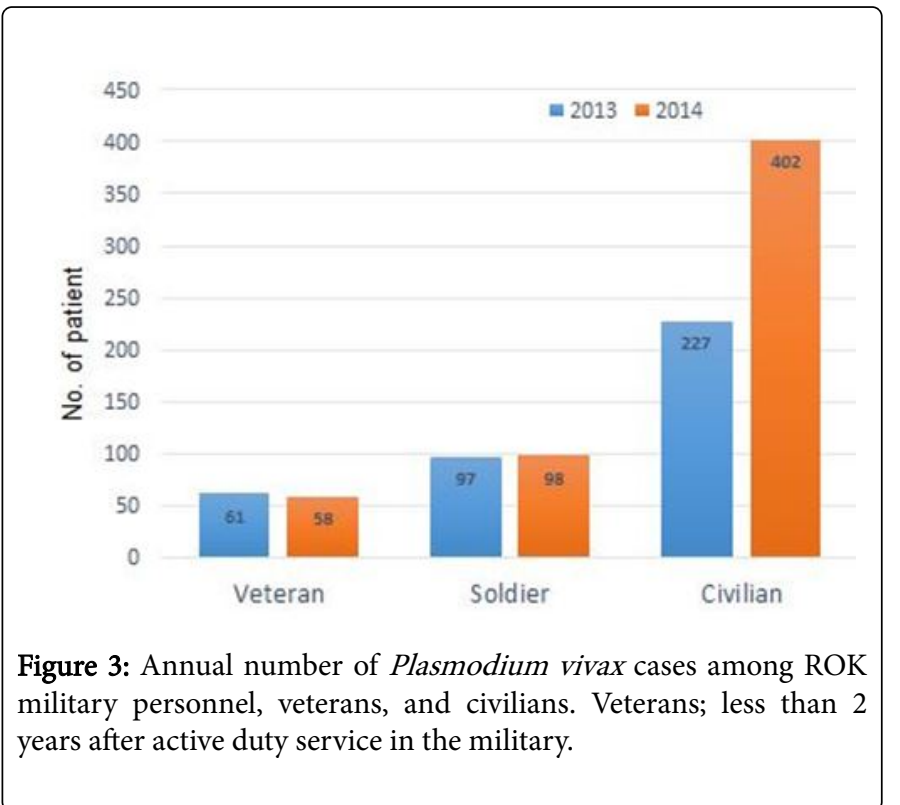

The annual proportion of patients in 16 administrative districts decreased by $30.6 \%$ in 2013 compared with that in 2012; however, in 2014, it increased by $44.9 \%$ compared with that in 2013 .

The number of cases reported in Seoul Metropolitan City, Incheon Metropolitan City, and Gyeonggi Province increased by 105.4\%, 57.7\%, and $41.7 \%$, respectively, in 2014 .

However, in Gangwon Province, the number of cases decreased by $40.0 \%$, from 25 cases in 2013 to 15 cases in 2014 (Table 1).

In addition, the US military reported 14 malaria cases during 2013-2014. Nine cases were reported in personnel currently deployed in Korea (one case in 2013 and eight cases in 2014), and five cases, two cases in 2013 and three cases in 2014 (unpublished data), were detected in US military personnel after their return to the United States, possibly owing to the long incubation period of vivax malaria.

Overall, the API decreased from 1.08 in 2012 to 0.75 in 2013 and then increased to 1.09 in 2014. In 2012, Incheon Metropolitan City had the highest API (4.93), followed by Gyeonggi Province (2.13), Gangwon Province (0.87), and Seoul Metropolitan City (0.66).

In 2013, Incheon Metropolitan City had the highest API (2.69), followed by Gyeonggi Province (1.65), Gangwon Providence (1.67), Gyeongbuk Province (0.41), and Seoul Metropolitan City (0.37). In 2014, Incheon Metropolitan City had the highest API (4.24), followed by Gyeonggi Province (2.34), Gangwon Province (1.00), Seoul Metropolitan City (0.75), and Chungnam Province (0.44) (Figure 4). 
Citation: $\quad$ Lee HW, Park SH, Na BK, Kim HC, Klein TA, Jeon BY, Shin EH, Bahk YY, Suh HH, Kim TS* and Chung MK* (2017) Changing Vivax Malaria Transmission Features in the Republic of Korea during 2013-2014. J Trop Dis 5: 231. doi:10.4172/2329-891X.1000231

Page 5 of 9

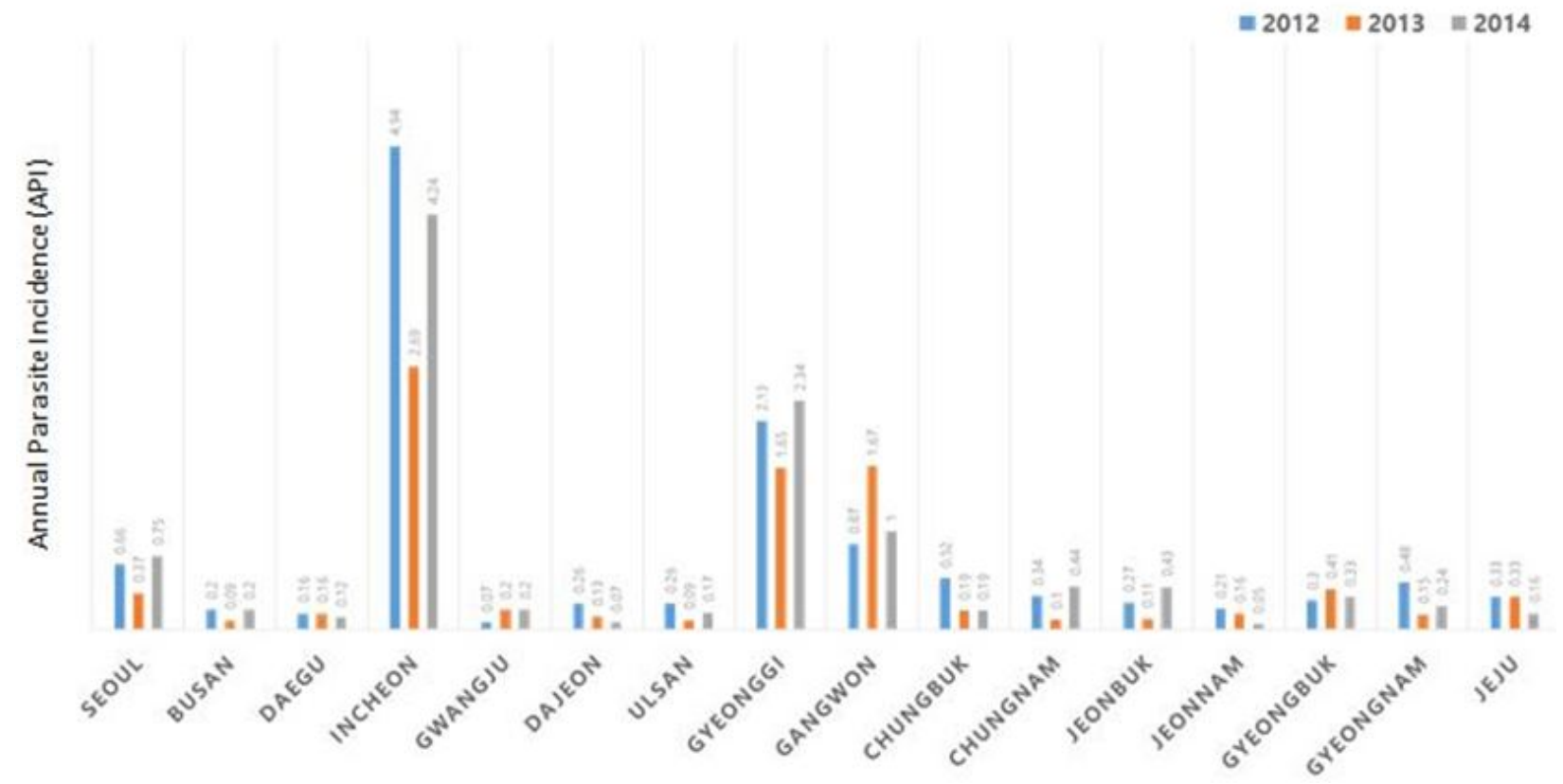

Figure 4: The annual parasite incidence (API) of Plasmodium vivax malaria patients from 2012 - 2014 according to metropolitan city and province.

Among the 943 total cases documented during a 2-year period, 847 (89.5\%) were reported in Incheon Metropolitan City (201 cases), Seoul Metropolitan City (113 cases), Gyeonggi Province (493 cases), and Gangwon Province (40 cases; Figure 5).

The US military reported 14 malaria cases during 2013-2014. Nine cases were reported in personnel currently deployed in Korea (one case in 2013 and eight cases in 2014), and five cases, two cases in 2013 and three cases in 2014 (unpublished data), were detected in US military personnel after their return to the United States, possibly owing to the long incubation period of vivax malaria.

Overall, the API decreased from 1.08 in 2012 to 0.75 in 2013 and then increased to 1.09 in 2014. In 2012, Incheon Metropolitan City had the highest API (4.93), followed by Gyeonggi Province (2.13), Gangwon Province (0.87), and Seoul Metropolitan City (0.66). Highrisk areas are also observed in the ROK adjacent to the malaria-risk areas in North Korea, such as Incheon Metropolitan City and northern Gyeonggi and Gangwon Provinces.

No objective opinion exists, that mass chemoprophylaxis with chloroquine (during malaria transmission season) and primaquine (after transmission season) would decrease the number of malaria cases among military personnel and may subsequently decrease the numbers of cases among civilian populations. Chemoprophylaxis started with approximately 16,000 military personnel in 1997, and has expanded to more than 200,000 army soldiers who are currently serving in areas with high risk for malaria.

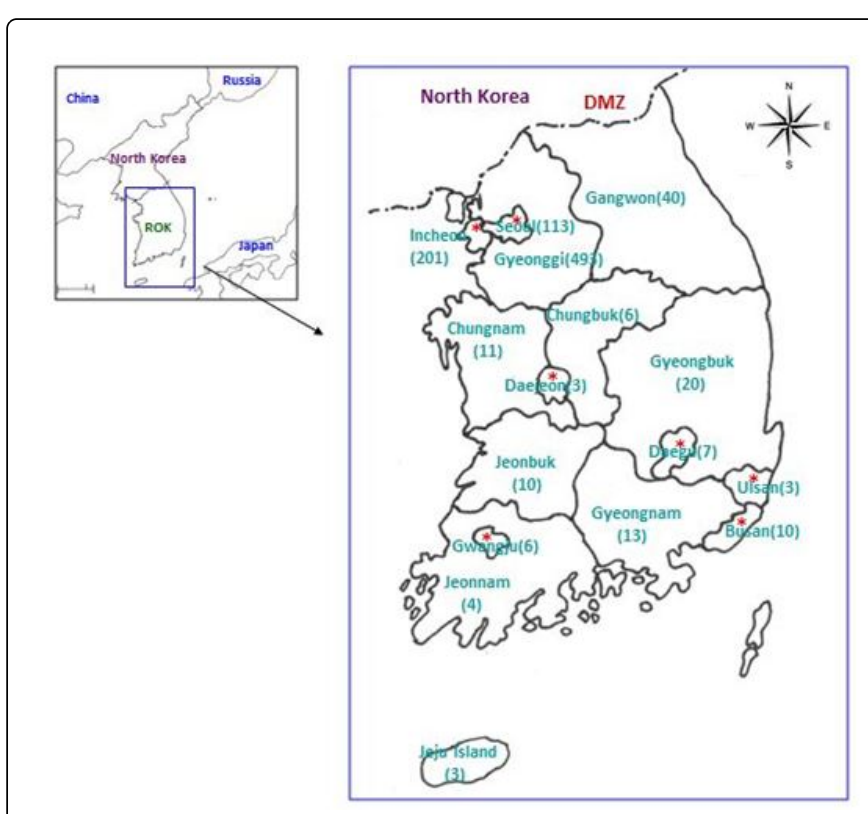

Figure 5: Map of the Republic of Korea showing provinces and metropolitan areas (indicated by asterisks) where malaria cases were reported from 2013 to 2014. DMZ, demilitarized zone.

From 2013 to 2014,755 men and 188 women had vivax malaria. The female-to-male ratio increased from 1:4.3 in 2013 to 1:3.8 in 2014. The ratio in 2014 was similar to that in 2012 (1:4.1; Figure 6). 
Citation: $\quad$ Lee HW, Park SH, Na BK, Kim HC, Klein TA, Jeon BY, Shin EH, Bahk YY, Suh HH, Kim TS* and Chung MK* (2017) Changing Vivax Malaria Transmission Features in the Republic of Korea during 2013-2014. J Trop Dis 5: 231. doi:10.4172/2329-891X.1000231

Page 6 of 9

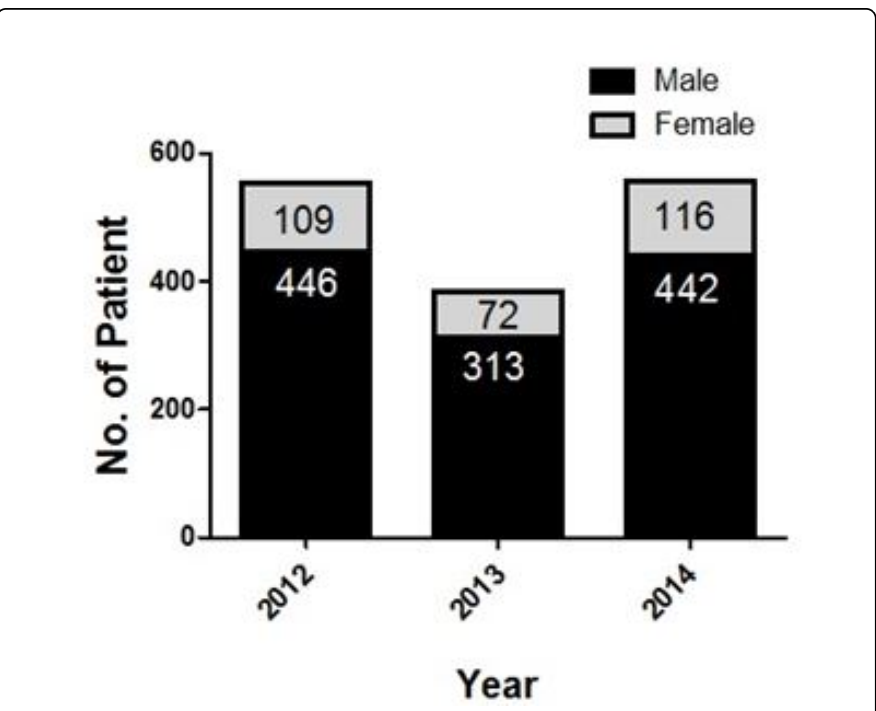

Figure 6: The sex ratio of patients with vivax malaria from 2013 to 2014.

Most malaria patients were soldiers $(21.80 \%$ in 2013 and $15.13 \%$ in 2014 ), followed by students ( $13.71 \%$ in $2013,8.17 \%$ in 2014$)$, clerks (5.84\% in $2013,4.69 \%$ in 2014$)$, and farmers and fishermen $(5.17 \%$ in $2013,3.93 \%$ in 2014; Figure 7).

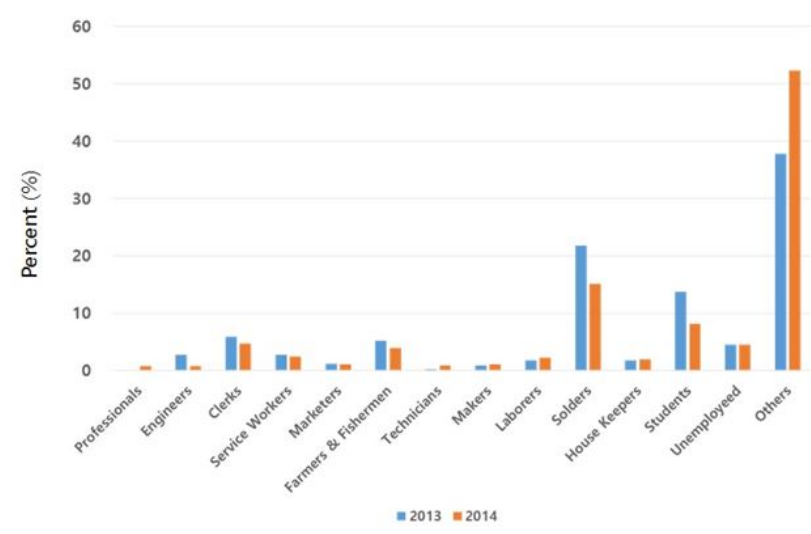

Figure 7: Occupations of patients with vivax malaria from 2013 to 2014.

According to age group, the largest number of patients were those aged 20-29 years (366 cases), followed by those aged 50-59 years (150 cases), 40-49 years (146 cases), and 30-39 years (125 cases). Unfortunately, one patient was aged $<5$ years (Figure 8 ).

High-risk areas are also observed in the ROK adjacent to the malaria-risk areas in North Korea, such as Incheon Metropolitan City and northern Gyeonggi and Gangwon Provinces. No objective opinion exists, that mass chemoprophylaxis with chloroquine (during malaria transmission season) and primaquine (after transmission season) would decrease the number of malaria cases among military personnel and may subsequently decrease the numbers of cases among civilian populations. Chemoprophylaxis started with approximately 16,000 military personnel in 1997, and has expanded to more than 200,000 army soldiers who are currently serving in areas with high risk for malaria.

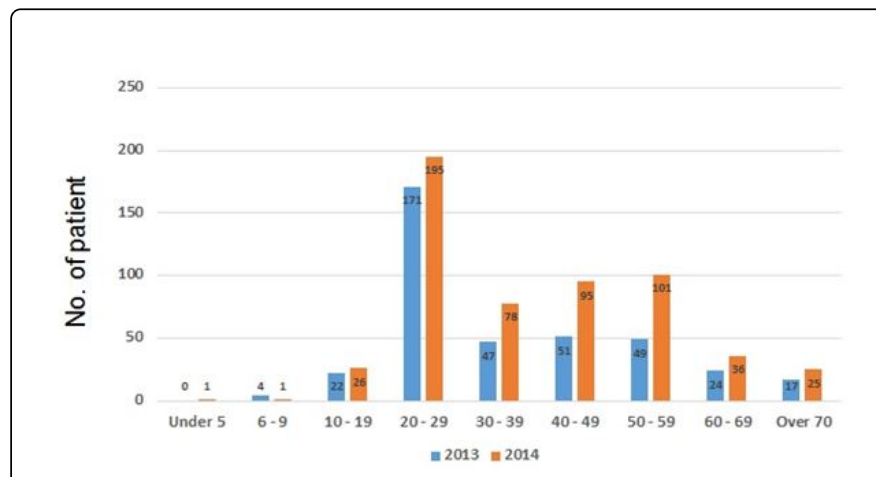

Figure 8: The number of Plasmodium vivax malaria patients according to patient age.

No cases of malaria were reported in January and December 2013 or in January and March 2014. The highest peaks were observed in July 2013 (91 cases) and 2014 (153 cases). More cases were reported through June 2013 than during the same period in 2014; however, the situation was reversed after July 2014. In particular, nine cases were reported in December 2014, whereas none was reported in December 2013 (Figure 9). In Gyeonggi Province, An. sinensis s.l. was first detected during the 24th week of 2013 and the 19th week of 2014 in Sau-dong, Gimpo-si and during the 20th week in both 2013 and 2014 in Daesung-dong, Paju-si. In Gangwon Province, it was first detected during the 24th week of 2013 and the 19th week of 2014 in Daema-ri, Cheorwon County; during the 24th week of 2013.

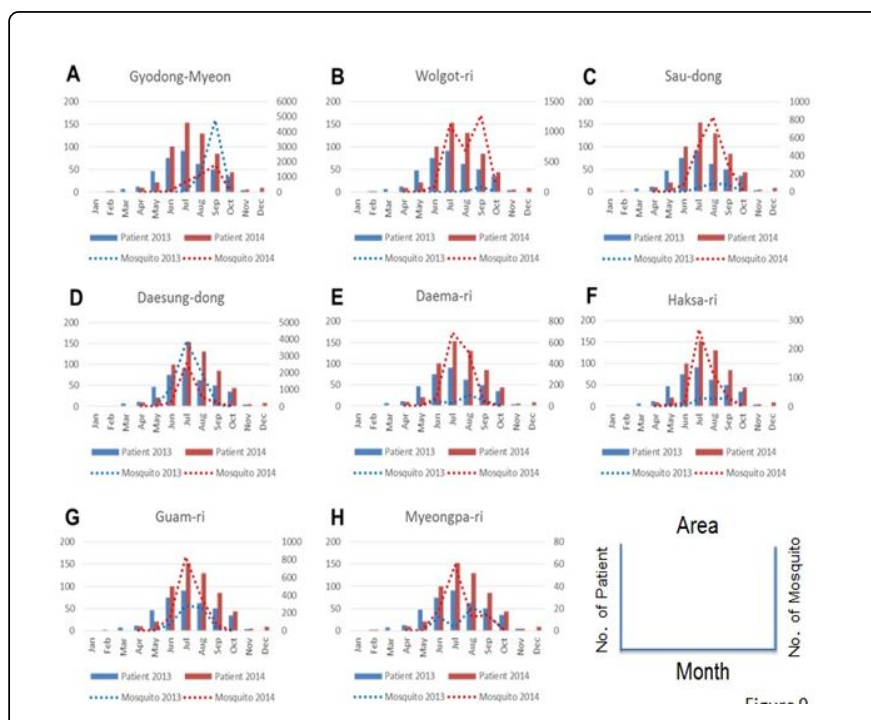

Figure 9: The monthly incidence of vivax malaria in the Republic of Korea and the number of Anopheles sinensis sensu lato collected from the malaria-risk areas in Incheon. Metropolitan city (A, B), Gyeonggi Province $(\mathrm{C}, \mathrm{D})$ and Gangwon Province $(\mathrm{E}-\mathrm{H})$ in the Republic of Korea from 2013 to 2014.

A total of 6,191 Anopheles sinensis sensu lato were collected in 2013, and 6,879 were collected in 2014 from sites in Gyodong-Myeon 
(Figure 9A) and Wolgot-ri (Figure 9B), Ganghwa County, Incheon Metropolitan City, from April to October. In Gyeonggi Province, 7,496 An. sinensis s.l. were collected in 2013, and 5,479 were collected in 2014 from Sau-dong (Figure 9C) and Daesung-dong (Figure 9D). In Gangwon Province, 1,033 An. sinensis s.l. were collected in 2013, and 3,264 were collected in 2014 from Daema-ri (Figure 9E), Haksa-ri (Figure 9F), Guam-ri (Figure 9G), and Myeongpa-ri (Figure 9H). Only two collection sites, Gyodong-Myeon in Incheon Metropolitan City and Josan-ri in Gyeonggi Province, showed higher mosquito densities in 2013; all other locations showed higher numbers of mosquitos in 2014.

An. sinensis s.l. was first detected during the 22 nd week of 2013 and the 20th week of 2014 in Gyodong-Myeon and during the 16th week of 2013 and the 14th week of 2014 in Wolgot-ri, Ganghwa County, Incheon Metropolitan City. In Gyeonggi Province, An. sinensis s.l. was first detected during the 24th week of 2013 and the 19th week of 2014 in Sau-dong, Gimpo-si and during the 20th week in both 2013 and 2014 in Daesung-dong, Paju-si. In Gangwon Province, it was first detected during the 24th week of 2013 and the 19th week of 2014 in Daema-ri, Cheorwon County; during the 24th week of 2013 and the 15th week of 2014 in Haksa-ri, Cheorwon County; during the 20th week of 2013 and the 19th week of 2014 in Guam-ri, Yanggu County; and during the 24th week of 2013 and the 23rd week of 2014 in Myeongpa-ri, Gosung County (Figure 9).

The highest density of An. sinensis s.l. was recorded during the 36th week $(3,584)$ of 2013 and the 36th week $(548)$ of 2014 in GyodongMyeon and during the 37th week (50) of 2013 and the 30th week (480) of 2014 in Wolgot-ri, Ganghwa County, Incheon Metropolitan City. In Gyeonggi Province, density peaked during the 35th week (40) of 2013 and the 33rd week (327) of 2014 in Sau-dong, Gimpo-si, and during the 28th week $(2,147)$ of 2013 and the 29th week $(983)$ of 2014 in Daesung-dong, Paju-si. In Gangwon Province, it peaked during the 33rd week (38) of 2013 and the 29th week (291) of 2014 in Daema-ri; during the 28th week (25) of 2013 and the 31st week (152) of 2014 in Haksari, Cheorwon County; during the 28th week (97) of 2013 and the 31st week (298) of 2014 in Guam-ri, Yanggu County; and during the 34th week (14) of 2013 and the 30th week (27) of 2014 in Myeongpa-ri, Gosung County (Figure 9).

\section{Discussion}

In North Korea, the high-risk areas for vivax malaria are Gaeseong City, South and North Hwanghae Provinces, and Gangwon Province, all of which border the DMZ. In general, no civilians have been allowed to enter the DMZ for more than 60 years, although there is one exception, the village of Daesung-dong, which is located inside the DMZ. The natural ecosystems and biodiversity are highly preserved in the DMZ [18]. Since 2002, North Korea has provided presumptive anti-relapse chemoprophylaxis with primaquine before the transmission season for civilians living in these areas to control malaria [19]. As a result, malaria transmission was believed to have occurred mainly in these areas in North Korea [20]. High-risk areas are also observed in the ROK adjacent to the malaria-risk areas in North Korea, such as Incheon Metropolitan City and northern Gyeonggi and Gangwon Provinces. No objective opinion exists, that mass chemoprophylaxis with chloroquine (during malaria transmission season) and primaquine (after transmission season) would decrease the number of malaria cases among military personnel and may subsequently decrease the numbers of cases among civilian populations. Chemoprophylaxis started with approximately 16,000 military personnel in 1997, and has expanded to more than 200,000 army soldiers who are currently serving in areas with high risk for malaria [21]. Owing to this effort, the ratio of civilian to soldier cases has changed completely compared with that observed early in the reemergence of malaria in Korea (Figure 3) [13]. This implies a trend of shifting to increasingly stable local transmission within these areas from malaria that was predominantly acquired in the DMZ (from across the border from North Korea).

During 2013-2014, most cases of malaria were reported from June through September, and the highest peaks were observed from the 27th week of 2013 (June 30 to July 6; 25 patients) and in the 31st and 32nd weeks of 2014 (July 27 to August 9; 44 patients each week). The second highest peak was observed in the 39th week of 2013 (September 22-28; 24 patients) after Chuseok (Korean Thanksgiving Day, September 19). Several more peaks were observed during the transmission season; in 2014, another peak was observed in the 38th week (September 14-20), again after Chuseok (September 8), with 27 patients. Interestingly, in the 41st week (early October) in both 2013 and 2014, the last peak of the transmission season was observed (15 patients in 2013 and 19 patients in 2014). The cases in veterans and soldiers were more stable than in civilians after 2012 (Figure 2); thus, the factors that affect the increase in the number of cases in 2014 compared with that in 2013 might be outdoor activities or travel of residents belonging to the age group 30-60 years (Figure 8) in areas with a higher chance of interacting with vector mosquitoes.

PHCs have the responsibility to control the transmission of malaria in each risk area. As a result of anti-malaria efforts by the PHC in Cheorwon County where there is a high-risk area in Gangwon Province, the number of malaria cases in 2014 decreased from 25 cases in 2013 (API: 1.67) to 15 cases (API: 1.00), which is close to same number of cases documented in 2012 (13 cases; API: 0.87; Figure 4). Interestingly, this province showed a reverse pattern in case numbers compared with that observed in other metropolitan cities and provinces (Incheon, Seoul, and Gyeonggi), where the numbers decreased in 2013 and increased in 2014 (Table 1). This may be because of the average duration until confirmation of suspected malaria cases, which was 5.12 days in 2014 but longer than that ( 7 days) in 2013 (unpublished data). The anti-mosquito treatments, including fogging and residual spraying, were performed by the PHC in Cheorwon County from May 1 to the end of October in both 2013 and 2014 (unpublished data). Larvicide was used eight times in 2013 and 10 times in 2014. Insecticide was applied 632 times in 2013 and 674 times in 2014 to cover an area of $36,592 \mathrm{~m}^{2}$ (data from the PHC in Cheorwon County, Gangwon Province).

\begin{tabular}{|l|l|l|l|l|}
\hline \multirow{2}{*}{ Month } & \multicolumn{3}{|l|}{$\mathbf{2 0 1 3}$} & $\mathbf{2 0 1 4}$ \\
\cline { 2 - 5 } & $\begin{array}{l}{ }^{*} \text { Temperature } \\
\left({ }^{\circ} \mathrm{C}\right)\end{array}$ & $\begin{array}{l}\text { **Precipitation } \\
(\mathrm{mm})\end{array}$ & $\begin{array}{l}\text { Temperature } \\
\left({ }^{\circ} \mathrm{C}\right)\end{array}$ & $\begin{array}{l}\text { Precipitation } \\
(\mathbf{m m})\end{array}$ \\
\hline $\begin{array}{l}\text { Spring } \\
\text { (Mar-May) }\end{array}$ & 11.6 & 264.3 & 13.1 & 215.9 \\
\hline $\begin{array}{l}\text { Summer } \\
(\text { Jun-Jul) }\end{array}$ & 25.4 & 567.5 & 23.6 & 599.8 \\
\hline $\begin{array}{l}\text { Fall } \\
(\text { Sep-Nov) }\end{array}$ & 14.6 & 231.2 & 14.9 & 293.1 \\
\hline $\begin{array}{l}\text { Winter } \\
\text { (Dec-Feb) }\end{array}$ & 1.5 & 59.7 & 0.7 & 76.7 \\
\hline
\end{tabular}




\begin{tabular}{|l|l|l|l|l|}
\hline $\begin{array}{l}\text { Annual } \\
\text { mean }\end{array}$ & 12.9 & $1,162.9$ & 13.1 & $1,173.5$ \\
\hline
\end{tabular}

Table 2: Comparison the variation of annual mean temperature and precipitation during 2013-2014.

Six Anopheles species were reported in the ROK: An. sinensis sensu stricto, Anopheles belenrae, Anopheles pullus, Anopheles kleini, Anopheles sineroides, and Anopheles lesteri [22-25]. These six species comprise the species complex An. sinensis s.l., and Anopheles collection data are presented as An. sinensis s.l. in this study. Analysis of the mosquito density of An. sinensis s.l. in the study areas over the 2-year study period showed that the density was higher in 2014 than in 2013 except in Gyodong-Myeon of Ganghwa County, Incheon Metropolitan City (Figure 9A), and Daesung-dong of Paju-si, Gyeonggi Province (Figure 9D). In addition, An. sinensis s.l. was first detected earlier in 2014 than in 2013.

The density of An. sinensis s.l. in more urbanized collection areas, such as Wolgot-ri of Ganghwa County, Incheon Metropolitan City (Figure 9B), and Sau-dong of Gimpo-si, Gyeonggi Province (Figure 9C), was relatively higher, and the malaria transmission season was longer.

Although the population density of Anopheles $s p$. increased at most of the collection sites in 2014, this was not the definitive reason for the increase in vivax malaria cases, as mosquito infectivity remains unclear. Infectivity for all six species should be investigated in future studies; to date infectivity analysis has been conducted for An. sinensis.

It was shown that the mean number of sporozoites in a single mosquito was $818(n=8$; range 648-1,056) when fed artificially with parasite-infected patient blood [26].

Interestingly, the annual mean temperature in 2014 was slightly higher than that in $2013\left(13.1^{\circ} \mathrm{C}\right.$ and $12.9^{\circ} \mathrm{C}$, respectively). The mean temperature during the summer season of 2014 was lower than that of 2013, whereas the mean temperature in the spring and autumn season of 2014 was higher than that of 2013 (Table 2) [27].

In addition, the annual mean precipitation in $2014(1,173.5 \mathrm{~mm})$ was slightly higher than that in $2013(1,162.9 \mathrm{~mm}$ ) (Table 2) [27]. Taking these observations together, it can be inferred that the increased number of patients in 2014 was closely related to elevated temperature, precipitation, and vector density.

\section{Conclusion}

The number of patients and API reported in 2014 (558 cases and 1.09 respectively) were similar to those in 2012 (555 cases and 1.08 respectively), despite the decrease in the number of cases in 2013 (385 cases and 0.75 , respectively). Although it is expected that the number of cases of malaria will continue to decrease some fluctuations and increases could occur over the next few years. Therefore, more intensive and systematic malaria surveillance is needed in the western part of Korea, especially in Incheon Metropolitan City and Gyeonggi Province, to reduce the potential for future increases in malaria cases in the ROK.

\section{Acknowledgements}

We are grateful to the staff of the Public Health Centers in Cheorwon, Paju, Gimpo, Ganghwa, Yanggu, and Gosung County and the Institutes of Health and Environment in Incheon Metropolitan City, Gangwon Province, and Gyeonggi Province. We thank Dr. Hun Jae Lee, Professor of Department of Social Medicine, Inha University School of Medicine for his help with statistics. This work was supported by a research grant from Inha University.

\section{References}

1. Mendis K, Sina BJ, Marchesini P, Carter R (2001) The neglected burden of Plasmodium vivax malaria. Am J Trop Med Hyg 64: 97-106.

2. Hasegawa (1913) Malaria in Korea. Chosen Igakkai Zasshi 4: 53-69.

3. National Malaria Eradication Service (1966) Ministry of Health and Social Affairs, ROK. Malaria pre-eradication programme in Korea. Progress report. pp: 44-70.

4. Paik YH, Rhee HI, Shim JC (1988) Malaria in Korea. J Exp Med 58: 55-66.

5. WHO (1981) Synopsis of the world malaria situation in 1979. Wkly Epidemiol Rec 56: 145-149.

6. Soh CT, Lee KT, Im KI, Min DY, Ahn MH, et al. (1985) Current status of malaria in Korea. Yonsei Rep Trop Med 16: 11-18.

7. Chai IH, Lim GI, Yoon SN, Oh WI, Kim SJ, et al. (1994) Occurrence of tertian malaria in a male patient who has never been abroad. Korean J Parasitol 32: 195-200.

8. Cho SY, Kong Y, Park SM, Lee JS, Lim YA, et al. (1994) Two vivax malaria cases detected in Korea. Korean J Parasitol 32: 281-284.

9. Park JW, Klein TA, Lee HC, Pacha LA, Ryu SH, et al. (2003) Vivax malaria: A continuing health threat to the Republic of Korea. Am J Trop Med Hyg 69: 159-167.

10. Lee JS, Kho WG, Lee HW, Seo M, Lee WJ (1998) Current status of vivax malaria among civilians in Korea. Korean J Parasitol 36: 241-248.

11. Klein TA, Pacha LA, Lee HC, Kim HC, Lee WJ, et al. (2009) Plasmodium vivax malaria among U.S. forces Korea in the Republic of Korea, 1993-2007. Mil Med 174: 412-418.

12. Kim HC, Pacha LA, Lee WJ, Lee JK, Gaydos JC, et al. (2009) Malaria in the Republic of Korea, 1993-2007. Variables related to re-emergence and persistence of Plasmodium vivax among Korean populations and U.S. forces in Korea. Mil Med 174: 762-769.

13. Yeom JS, Ryu SH, Oh S, Lee WJ, Kim TS, et al. (2005) Status of Plasmodium vivax malaria in the Republic of Korea during 2001-2003. Am J Trop Med Hyg 73: 604-608.

14. Jun G, Yeom JS, Hong JY, Shin EH, Chang KS, et al. (2009) Resurgence of Plasmodium vivax malaria in the Republic of Korea during 2006-2007. Am J Trop Med Hyg 81: 605-610.

15. Yeom JS, Jun G, Kim JY, Lee WJ, Shin EH, et al. (2012) Status of Plasmodium vivax malaria in the Republic of Korea, 2008-2009: decrease followed by resurgence. Trans R Soc Trop Med Hyg 106: 429-436.

16. Moody A (2002) Rapid diagnostic tests for malaria parasites. Clin Microbiol Rev 15: 66-78.

17. McKenzie FE, Prudhomme WA, Magill AJ, Forney JR, Permpanich B, et al. (2005) White blood cell counts and malaria. J Infect Dis 192: 323-330

18. Kim KC (1997) Preserving biodiversity in Korea's demilitarized zone. Science 278: 242-243.

19. Korea Centers for Disease Control and Prevention (2005) Effectiveness and safety of primaquine chemoprophylaxis against P. vivax before transmission season. Report of Bi-regional Meeting for Control of Malaria in East Asia 8: 17-20.

20. Yeom JS, Kim TS, Oh S, Sim JB, Barn JS, et al. (2007) Plasmodium vivax malaria in the Republic of Korea during 2004-2005: changing patterns of infection. Am J Trop Med Hyg 76: 865-868.

21. Lee KS, Kim TH, Kim ES, Lim HS, Yeom JS, et al. (2009) Chloroquineresistant Plasmodium vivax in the Republic of Korea. Am J Trop Med Hyg 80: 215-217. 
Citation: $\quad$ Lee HW, Park SH, Na BK, Kim HC, Klein TA, Jeon BY, Shin EH, Bahk YY, Suh HH, Kim TS* and Chung MK* (2017) Changing Vivax Malaria Transmission Features in the Republic of Korea during 2013-2014. J Trop Dis 5: 231. doi:10.4172/2329-891X.1000231

Page 9 of 9

22. Rueda LM (2005) Two new species of Anopheles (Anopheles) Hyrcanus group (Diptera: Culicidae) from the Republic of South Korea. Zootaxa 941: 1-26.

23. Tanaka K, Mizusawa K, Saugstad ES (1979) A Revision of the adult and larval mosquitoes of Japan (including the Ryukyu Archipelago and the Ogasawara Islands) and Korea (Diptera: Culicidae). Contrib Am Entomol Inst 16: 1-987.

24. Hwang UW (2007) Revisited ITS2 phylogeny of Anopheles (Anopheles) Hyrcanus Group mosquitoes: Reexamination of unidentified and misidentified ITS2 sequences. Parasitol Res 101: 885-894.
25. Li C, Lee JS, Groebner JL, Kim HC, Klein TA, et al. (2005) A newly recognized species in the Anopheles Hyrcanus Group and molecular identification of related species from the Republic of South Korea (Diptera: Culicidae). Zootaxa 939: 1-8.

26. Lee HW, Cho SH, Shin EH, Lee JS, Lee JS, et al. (2001) Experimental infection of Anopheles sinensis with Korean isolates of Plasmodium vivax. Korean J Parasitol 39: 177-183.

27. Kim TS, Kim JS, Na BK, Lee WJ, Kim HC, et al. (2013) Decreasing incidence of Plasmodium vivax in the Republic of Korea during 2010-2012. Mala J 12: 309. 